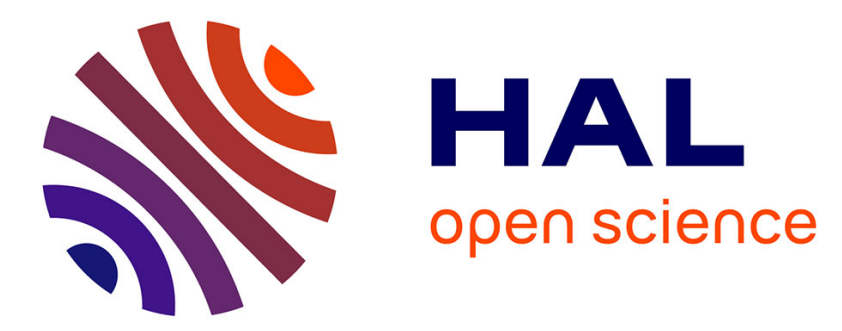

\title{
Comment construire les proximités entre stations de moyenne montagne grâce à un objet-frontière? L'exemple de la charte de développement durable
}

Rachel Bocquet, Caroline Mothe

\section{- To cite this version:}

Rachel Bocquet, Caroline Mothe. Comment construire les proximités entre stations de moyenne montagne grâce à un objet-frontière? L'exemple de la charte de développement durable. Management international, A paraître, 22 (4), pp.27-38. hal-01695593

\section{HAL Id: hal-01695593 \\ https://hal.univ-smb.fr/hal-01695593}

Submitted on 30 Jan 2018

HAL is a multi-disciplinary open access archive for the deposit and dissemination of scientific research documents, whether they are published or not. The documents may come from teaching and research institutions in France or abroad, or from public or private research centers.
L'archive ouverte pluridisciplinaire HAL, est destinée au dépôt et à la diffusion de documents scientifiques de niveau recherche, publiés ou non, émanant des établissements d'enseignement et de recherche français ou étrangers, des laboratoires publics ou privés. 


\title{
Comment construire les proximités entre stations de moyenne montagne grâce à un
}

\section{objet-frontière ? L’exemple de la charte de développement durable}

\author{
Rachel Bocquet ${ }^{1}$ et Caroline Mothe ${ }^{1}$
}

${ }^{1}$ IREGE, Université Savoie Mont Blanc

Mots-clés : Charte, objet-frontière, proximités, gouvernance territoriale, montagne.

Keywords: Chart, boundary object, proximities, territorial governance, mountains.

\section{Résumé}

L'objectif de cette recherche est de déterminer si et comment un objet-frontière peut participer à la construction des proximités nécessaires à la gouvernance territoriale en stations de montagne. L'étude qualitative porte sur la création d'une charte de développement durable entre trois stations. Les résultats montrent, qu’au-delà de la proximité géographique entre les stations, la charte a permis de créer une proximité institutionnelle et de dépasser des relations entre les acteurs régies jusqu'ici davantage par le mode de l’indifférence ou de la confrontation. Les implications théoriques et managériales dans le cadre de la mise en place d’une gouvernance territoriale inter-stations sont discutées. 
Les stations de montagne, et notamment celles dites moyennes ${ }^{1}$, sont confrontées aux enjeux majeurs de la diversification quatre saisons en réponse à l'évolution des demandes touristiques, au réchauffement climatique et à la raréfaction de l’or blanc. Aussi l'action publique a-t-elle développé depuis les années 2000 un ensemble de dispositifs incitatifs et d'accompagnement des stations de moyenne montagne, que ce soit au niveau régional (avec les conventions Stations Durables depuis 2010) ou à l'échelle du massif des Alpes (avec les conventions Espaces Valléens). Ces dispositifs permettent à des stations proches, géographiquement parlant, de se coordonner pour mener des actions notamment liées à la durabilité des modèles touristiques et à une offre renouvelée qui permet d'attirer une clientèle tout au long de l'année. Ils ont tendance à modifier le champ d'action des gouvernances locales, incitant à la mise en place d'une gouvernance territoriale autour de diverses proximités qu’il convient par conséquent de créer, ou de renforcer.

La question qui se pose alors est de savoir comment les acteurs s’approprient ces nouveaux cadres d'actions et de structuration de l'espace pour mener les actions collectives. Nous interrogeons ici cette coordination entre acteurs et la manière dont lesdits acteurs peuvent construire les proximités nécessaires à la mise en place d’une gouvernance territoriale. L’économie de la proximité reconnaît différentes formes de proximité, discutées au sein de deux approches. D’une part, l'approche «interactionniste » met l'accent, au-delà de la proximité géographique (spatiale), sur une autre forme de proximité d'essence cognitive, qualifiée de proximité organisée (Rallet et Torre, 2005). Elle souligne la capacité des acteurs géographiquement proches et partageant un même référentiel cognitif à mener des projets collaboratifs. D’autre part, l'approche dite «institutionnaliste » considère trois formes de proximité, géographique, organisationnelle et institutionnelle (Talbot, 2008 ; Colletis et

\footnotetext{
${ }^{1}$ Les stations de moyenne montagne, situées entre 600 et 900-2200 mètres d'altitude (selon les massifs), sont fragilisées par le déficit chronique d'enneigement. Si ces stations possèdent des conditions propices au développement de stratégies de diversification quatre saisons basée sur les activités de pleine nature (forêts, alpages), elles tardent encore à les mettre en œuvre (ANEM, 2013).
} 
Pecqueur, 2005) et introduit explicitement le rôle des institutions. A la différence de l'approche interactionniste, qui ne nie pas l'existence de conditions politiques à la coordination mais s'y attarde peu, elle considère que les conditions de la coordination ne sont pas seulement d'ordre cognitif mais aussi d'ordre politique (Talbot, 2008).

Si les proximités jouent un rôle clé pour la gouvernance territoriale (Achin, 2015 ; Rallet et Torre, 2004; Zimmerman, 2008), en particulier les proximités géographique, organisationnelle et institutionnelle (Gilly et Wallet, 2005 ; Leloup, Moyart et Pecqueur, 2005), leur processus de construction reste toutefois peu étudié (Balland, Boschma et Frenken, 2015). Or, la proximité géographique est loin d'être suffisante (François et Marcelpoil, 2008 ; Rallet et Torre, 2004 ; Zimmerman, 2008). En effet, bien qu'une telle proximité soit vue comme un vecteur de coopération et de transfert de savoirs et compétences, d'autres formes de proximité s’avèrent nécessaires pour qu’un apprentissage entre acteurs puisse avoir lieu (Boschma, 2005) et pour conduire une véritable action collective (Talbot, 2008 ; Colletis et Pecqueur, 2005). Dans une approche institutionnaliste, ces proximités restent à construire. La question « comment créer de telles proximités pour mettre en place une gouvernance territoriale ? », traitée par Gilly et Wallet (2005), mérite d’être approfondie.

Pour combler ce vide, nous nous appuyons sur le concept d'objet-frontière qui possède des propriétés permettant de résoudre les problèmes de coordination entre acteurs très hétérogènes et de créer par là-même cet espace de coordination nécessaire à la construction des proximités. Par définition, l’objet-frontière, qu’il soit tangible ou intangible, lie les représentations entre des acteurs ayant des horizons différents dans le but de servir un objectif commun (Star et Griesemer, 1989). L’objet-frontière est un concept particulièrement intéressant à mobiliser dans notre cadre d'analyse car il est un espace permettant la communication entre des acteurs ayant des horizons très différents afin de servir un objectif commun. Tout son intérêt réside dans le fait qu'il permet de mieux analyser les obligations de 
négociation et d’organisation entre des acteurs travaillant sur des dispositifs communs mais ayant a priori chacun leurs représentations propres.

Nous cherchons donc à comprendre comment un objet-frontière peut créer et renforcer lesdites proximités. Notre question de recherche est donc la suivante : « comment un objetfrontière permet-il de construire les proximités nécessaires à la mise en place d'une gouvernance territoriale ?».

L'originalité de cette recherche réside dans la combinaison du concept de proximités avec celui, plus largement utilisé en sociologie, d'objet-frontière. En mobilisant l'approche institutionnaliste des proximités, nous nous intéressons ici à leur construction via un objetfrontière, contrairement à la majorité des travaux qui se focalise sur les effets desdites proximités sur les interactions entre les acteurs.

La première partie de l'article s’attache à combiner deux approches théoriques, celle sur les proximités (dans une perspective institutionnaliste) en lien avec la gouvernance des stations et celle sur les objets-frontières. La deuxième partie, empirique, analyse qualitativement, à partir de données riches, primaires et secondaires, comment l’objet-frontière que représente la création d'une charte de développement durable entre les communes haut-savoyardes de Combloux, Praz-sur-Arly et Cordon en France, a permis de renforcer les proximités entre ces trois stations de montagne. Dans une dernière partie, nous discutons les résultats obtenus et concluons en formulant des implications et recommandations, notamment managériales, et en proposant des pistes de recherches futures. 


\section{PROXIMITES ET OBJET-FRONTIERE POUR LA MISE EN PLACE D'UNE GOUVERNANCE TERRITORIALE}

Après avoir introduit la notion de gouvernance territoriale et présenté l'approche institutionnaliste des proximités, nous montrerons en quoi l’objet-frontière peut jouer le rôle de support desdites proximités. La définition retenue de la gouvernance territoriale est la suivante: « un processus non seulement de coordination entre acteurs mais aussi d'appropriation des ressources et de construction de la territorialité [...] qui repose sur la multiplicité d'acteurs, la définition d'un espace identitaire et l'élaboration d'actions, de productions communes. Cette forme particulière de gouvernance sous-tend une proximité multiple puisqu'elle combine proximité géographique et proximité organisationnelle et institutionnelle des acteurs » (LELOUP, MOYART et PECQUEUR, 2005, p.330). Si cette définition a le mérite de mettre clairement en avant les différentes proximités qui la soustendent, elle ne permet pas de comprendre la manière dont celles-ci sont créées.

\section{Proximités géographique, organisationnelle et institutionnelle : le cadre institutionnaliste}

Le terme «proximité » a fait l’objet de nombreux développements, en particulier au sein de l'école française et de ses deux courants « interactionniste » et «institutionnaliste ». Alors que le premier reconnaît les deux formes de proximité que sont la proximité géographique et la proximité organisée (Torre et Rallet, 2005), le second décompose la proximité en trois formes distinctes et complémentaires : la proximité géographique, organisationnelle et institutionnelle. Il ne s’agit pas là d’une simple différence de terminologie. Derrière ces deux typologies, se manifeste une conception distincte de la coordination et de l'action collective (Talbot, 2008). En effet, pour le courant interactionniste, au-delà de la proximité géographique (physique), la coordination entre les acteurs est associée à un phénomène essentiellement cognitif basé sur deux logiques de proximité organisée. D’une part, une logique d'appartenance au sein de laquelle «deux membres d'une organisation sont proches 
l'un de l'autre parce qu'ils interagissent et que leurs interactions sont facilitées par des règles et routines de comportement (explicites ou implicites) qu'ils suivent » (Torre et Rallet, 2005, p. 49-50). D’autre part, une logique de similitude où «les membres d'une même organisation sont réputés partager un même système de représentations, ou ensemble de croyances, et les mêmes savoirs » (Torre et Rallet, 2005, p. 50).

Or, cette conception présente une limite principale que les tenants de l'approche institutionnaliste se sont attachés à rendre compte (cf. Talbot, 2008 pour une synthèse), notamment dans le cadre de la gouvernance territoriale ou locale (Gilly et Wallet, 2005 ; Leloup, Moyard et Pecqueur, 2005). Cette limite concerne la non prise en compte explicite des institutions comme condition sine qua non à la coordination entre les acteurs (Talbot, 2008). D’un point de vue institutionnaliste, il convient donc de considérer le rôle des institutions comme un cadre nécessaire à l'action collective en faisant exister une autre forme de proximité : la proximité institutionnelle. Cette dernière suppose la création d'institutions qui ne sont pas extérieures aux acteurs mais bien l'œuvre d'une intentionnalité collective. Elle permet de révéler des significations communes aux acteurs et de fixer les rôles complémentaires à jouer dans l’action collective (Talbot, 2008).

Cette forme de proximité institutionnelle qui consiste donc «à partager des idées communes et à assumer des rôles complémentaires " (Talbot, 2008, p. 306) s'avère intéressante là où l’hétérogénéité des acteurs est particulièrement élevée, contraignant ainsi à la coordination. Elle suppose la création d'un cadre commun qui ne fournit pas seulement « une vision partagée du monde » mais « une dimension politique régulatrice qui attribue des rôles à des acteurs hétérogènes afin d'apaiser transitoirement les conflits » (Talbot, 2008, p. 301). Nous comprenons dès lors toute la portée de cette forme de proximité pour la mise en place d'une gouvernance territoriale entre différentes stations de montagne qui n’appartiennent pas a priori à une même organisation. Certains auteurs ont d'ailleurs déjà 
bien révélé cette difficulté à l'échelle d’une station en mettant en évidence l’hétérogénéité des acteurs et la pluralité de systèmes productifs particuliers (entreprises indépendantes, remontées mécaniques etc.) (Bocquet, 2008 ; Marcelpoil, 2012 ; Gerbaux et Marcelpoil, 2006).

Les difficultés de coordination entre acteurs hétérogènes sont renforcées compte tenu du changement d'échelle, désormais inter-stations. En l'absence de structure particulière préexistante, les acteurs ne peuvent se rapprocher grâce à un référentiel de représentations et de connaissances communes, autrement dit grâce à un référentiel cognitif commun (Boschma, 2005). Dès lors, la proximité institutionnelle permet aux acteurs de se doter d'un cadre commun contraignant capable de réguler leurs actions pour atteindre les objectifs fixés. Dans la lignée de Talbot (2008), nous considérons que la proximité organisationnelle apparaît comme une forme particulière de proximité institutionnelle au sein de laquelle les acteurs se sont dotés d'une organisation, de règles de fonctionnement et d'une structure de pouvoir pour mener l'action collective.

Toutefois, il reste à comprendre comment se créent ces proximités entre les stations de montagne. La majorité des travaux restant théoriques et statiques (Boudières et Marcelpoil, 2006 ; Talbot, 2008), nous cherchons à aller plus loin en identifiant le rôle joué par l'objetfrontière.

\section{L'objet-frontière, un objet créateur de proximités}

Le concept d'objet-frontière est défini par les auteurs fondateurs, nous l'avons vu, comme un objet qui lie les représentations entre des acteurs appartenant à des mondes sociaux ${ }^{2}$ différents dans le but de servir un objectif commun (Star et Griesemer, 1989). Ce concept

\footnotetext{
${ }^{2}$ «Les mondes sociaux se réfèrent à des groupes d'activité n'ayant ni frontière claire ni organisation formelle et stable. Ils se constituent au travers de la relation entre les interactions sociales qui dérivent de l'activité primaire et la définition de la réalité pertinente. La notion relève du courant de l'interactionnisme symbolique » (Vinck, 2009, p. 53).
} 
s’avère particulièrement intéressant pour faire émerger la coopération ou pour étudier les pratiques coopératives entre des milieux variés. Le terme d’objet-frontière est apparu pour la première fois dans une étude sur les mécanismes de coordination du travail scientifique (Star et Griesemer, 1989). Dans cette recherche, les auteurs montrent comment un chercheur a pu faire coopérer des acteurs d’horizons disparates (administrateurs de l’université, conservateurs du patrimoine, collectionneurs amateurs, trappeurs, fermiers, etc.) autour de la création du Musée de Zoologie Vertébrée de l'Université de Californie. Les acteurs ont réussi à se comprendre, à partager leurs points de vue et à coopérer autour d'objets, tout en conservant leur identité propre. La coordination n’a pu être efficiente qu’au prix d’une « réconciliation » autour de la signification d'une même chose dans des mondes différents.

Les auteurs, en observant comment ces mondes socio-économiques hétérogènes collaborent et communiquent, ont montré que cette coopération se réalise autour d' « objets » communs ou « frontières », qui peuvent revêtir différents types ${ }^{3}$ (Trompette et Vinck, 2009), rendant ainsi l'objet-frontière «multiple ». Quel que soit le type de l'objet-frontière, ils montrent que le travail mobilisant des acteurs ayant des intérêts divergents requiert une importante, inévitable et indispensable coopération, qui sera l’objet d'une tension entre points de vue divergents. Par ailleurs, cette coopération n’existera que si un travail de « traduction ${ }^{4}$ » des divergences est réalisé.

Ce concept, principalement utilisé dans le monde scientifique (Star et Griesemer, 1989), a été appliqué dans des domaines très divers tels que la sociologie (Weedman, 1998), le management des connaissances (Wenger, 2000) ou encore l'innovation (Swan et al., 2002,

\footnotetext{
${ }^{3}$ Trompette et Vinck (1989, p. 8) identifient quatre types d'objets-frontières : 1) l'objet malléable qui peut être façonné par chacun ; 2) l'objet bibliothèque dont chacun peut extraire ce dont il a besoin ; 3) l'objet qui peut être simplifié (abstraction) ou dont on ignore les propriétés dont on n'a pas besoin ; 4) l'interface ou standard d'échange.

${ }^{4}$ Bien qu'ils reprennent la notion de traduction de Callon (1986), Star et Griesemer (1989) s'en échappent. En s'inscrivant dans une lecture plus écologique, ils cherchent à rompre avec la lecture asymétrique des opérations de traduction qui conduit à privilégier la prééminence de certains acteurs sur d’autres.
} 
2007) ${ }^{5}$. Nous l'utilisons ici pour la construction des proximités entre différentes stations de montagne au sein d'un territoire. L'objet-frontière répond à plusieurs caractéristiques pour favoriser la communication, la réalisation d'un objectif commun, la négociation et l'organisation entre des mondes très différents. Il est composé de plusieurs parties et sa modularité peut servir de base de dialogue entre ces différents mondes, dans différentes situations. Son caractère général oblige à un certain niveau d'abstraction pour faciliter le dialogue et assurer un minimum d'identité commune tout en étant suffisamment souple pour s’adapter aux besoins et contraintes spécifiques de chacun de ces mondes (Star, 1999). Sa polyvalence peut garantir son utilisation dans plusieurs activités, plusieurs pratiques dans le temps et l'espace. Sa standardisation garantit que l'information qu'il contient puisse être fournie sous une forme directement interprétable pour être utilisée localement. Finalement, pour qu'un objet devienne un objet-frontière, il faut qu'il y ait à la base une intention qui enclenche un processus de construction et/ou de reconnaissance, de désignation d'un objet comme étant un objet-frontière.

Le concept est utilisé pour décrire comment les acteurs maintiennent leurs différences et leur coopération, comment ils gèrent et restreignent la variété, comment ils se coordonnent dans le temps et l'espace. Il qualifie la manière dont les acteurs peuvent négocier leurs différences et créer une mise en accord de leurs points de vue respectifs. Ces caractéristiques de l'objet-frontière sont particulièrement utiles pour leur portée analytique (Trompette et Vinck, 2009) et pour comprendre comment il peut opérer en tant que support clé de la construction des proximités.

D'une part, l'objet-frontière « est un objet suffisamment flexible pour s'adapter aux besoins et aux nécessités propres des différents acteurs qui l'utilisent et qui sont suffisamment robustes pour maintenir une identité commune » (Star et Griesemer, 1989, p. 393). Qu'il soit tangible

\footnotetext{
${ }^{5}$ Pour une présentation détaillée des utilisations très variées et multiples exemples du concept d'objet-frontière, le lecteur est invité à se reporter à l'article de synthèse de Trompette et Vinck (2009).
} 
ou intangible, abstrait ou concret, universel ou spécifique, matériel ou conceptuel, cette propriété de «flexibilité interprétative » est aujourd’hui largement acceptée comme support de traductions hétérogènes, dispositif d’intégration des savoirs ou encore comme médiation dans les processus de coordination entre experts et non experts (Trompette et Vinck, 2009). En ce sens, l'objet-frontière permettrait d'être un support à la dimension cognitive de la proximité institutionnelle.

D’autre part, l'objet-frontière possède une seconde propriété, celle d'incorporer une infrastructure invisible à travers l'existence d'un ensemble de conventions, de standards, de normes indexées à une communauté de pratiques. Cette infrastructure est invisible au sens de Berry (1983) car elle n’est pas seulement un moyen de gérer au service de l'action (valeur technique) mais aussi un moyen d'articuler des rapports de force entre des individus et entre des groupes sociaux (valeur institutionnelle). Cette propriété permet notamment de rendre compte des processus de délégation d'activité et de fixation des rôles (Trompette et Vinck, 1989). Elle est particulièrement pertinente pour appréhender la dimension politique de la proximité institutionnelle. L'objet-frontière est un outil de compréhension et permet ainsi une multitude de coopérations et de coordinations entre les acteurs en créant une base commune tout en cadrant les agissements des acteurs (Star et Griesemer, 1989).

L'objet-frontière, pertinent pour examiner les pratiques collaboratives entre acteurs hétérogènes, l'est donc aussi comme support à la proximité institutionnelle (dans ses dimensions tant cognitive que politique) dans le sens où, nous l'avons vu, il contribue à l'identification de significations communes et fournit ainsi le cadre de la coordination cognitive entre les acteurs. En accompagnant les actions de chacun, il permet aussi une coordination politique entre les acteurs en régulant d'éventuels conflits. Cet objet-frontière, 
mis en place de manière intentionnelle, peut être assimilé à un objet institué ${ }^{6}$ qui rend possible l'action collective et participe ainsi à la mise en place d'une gouvernance territoriale. Dans la partie empirique, nous montrons comment un tel objet-frontière, en l'occurrence une charte de développement durable, a permis la création d’une proximité institutionnelle. Le tableau 1 ciaprès propose une synthèse de la nature de l'objet-frontière.

\section{[Insérer ici Tableau 1]}

\section{LA CHARTE DE DEVELOPPEMENT DURABLE ENTRE TROIS STATIONS DE MONTAGNE}

Nous analyserons ici comment la charte de développement durable à l'échelle de trois communes en Haute-Savoie (Combloux, Cordon et Praz-sur-Arly) a, en tant qu'objetfrontière, eu un rôle déterminant dans la construction de la proximité institutionnelle entre les acteurs.

La notion de charte de développement durable a été introduite dans les années 2000 par l'association Mountain Riders et reprise par l'Association Nationale des Maires de Stations de Montagne (ANMSM) en 2007. L'objectif de cet accord dans lequel se sont engagées 51 stations de montagne françaises, est d'aider à prendre en compte et à concilier les trois dimensions économique, sociale et environnementale du développement durable dans leurs politiques locales, au-delà des obligations réglementaires. La charte est construite autour de 8 plans d'actions et de 130 engagements qui abordent des thématiques variées telles que l'eau, l’énergie, les transports, la sensibilisation à l'environnement, l'activité touristique, les déchets, la préservation des paysages et des espaces naturels, l’habitat et l'aménagement durable.

\footnotetext{
${ }^{6}$ Nous remercions l'un de nos rapporteurs pour cette qualification de l'objet-frontière qui permet de rendre compte plus explicitement de son rôle régulateur, capable en cela de faire la jonction entre plusieurs organisations même s'il peut s'y manifester de manière singulière dans chaque lieu (Berry, 1983)
} 


\section{Contexte de la recherche}

La charte de développement durable mise en place entre les trois stations s’inscrit dans un cadre plus vaste composé d'un ensemble de dispositifs situés à différents échelons.

\section{Au niveau européen et du massif des Alpes}

La convention Espace Valléen ${ }^{7}$ est un programme d'aménagement durable qui propose des aides techniques et financières (fonds européens) pour aider des projets à l'échelle de vallées ou de territoires pertinents et permettre l'approfondissement des actions en matière de compétences touristiques. Le territoire qui bénéficie du «label» Espace Valléen peut solliciter plusieurs co-financeurs, notamment l’Europe à travers le Programme Opérationnel Interrégional Alpin (POIA), la Région et l'Etat à travers la Convention Interrégionale du massif des Alpes (CIMA) pour les projets de diversification touristique ou pour des problématiques liées à l’hébergement touristique et à la mobilité.

\section{Au niveau national}

Le lancement du Plan Neige en 1964, programme d'aménagement des stations de montagne, marque le désir de contrôler nationalement le développement local du tourisme de sport d'hiver. Le rôle de l'Etat dans la construction et l'essor des stations fut important de 1965 à 1975. Durant cette période, un programme de constructions immobilières (appartements et résidences secondaires) a été réalisé afin que les stations développent leurs domaines skiables et puissent financer leurs investissements en remontées mécaniques. Les stations souhaitées et créées par le Plan Neige ont permis le développement d'une véritable industrie touristique en territoires de montagne.

Les années 1980 laissent place à la décentralisation et à la Loi Montagne (1985) qui vise au développement et à la protection de la montagne. Il s'agit désormais de mettre en place un

\footnotetext{
${ }^{7}$ http://www.ccpmb.fr/les-projets-pour-le-territoire/contrats-regionaux-et-europeens/les-projets-europeens.
} 
projet territorial et une croissance «harmonisée » de la montagne valorisant davantage le caractère exceptionnel de la montagne aux niveaux patrimonial, culturel et économique. La loi Montagne indique également les précautions à prendre en termes d'urbanisation et d’équipement en montagne qui doivent respecter le milieu montagnard.

Par ailleurs, dans le cadre de la loi NOTRe (Loi n²015-991 du 7 août 2015 portant nouvelle organisation territoriale de la République ${ }^{8}$ ), les compétences des régions se voient renforcées et les compétences attribuées à chaque collectivité territoriale sont redéfinies précisément. Cette loi s'accompagne également d'une rationalisation de l'organisation territoriale avec la mise en place de nouveaux dispositifs visant à encourager les intercommunalités. A travers ces différentes législations, c’est l’importance d’une coopération entre stations de montagne qui est reconnue - et qui reste largement à mettre en place.

Au niveau régional

La Région Rhône-Alpes a, depuis la fin des années 90, mis en place un ensemble de dispositifs d'accompagnement des stations de moyenne montagne avec les contrats Stations Moyenne Montagne des années 2000 (dont avait bénéficié Praz sur Arly entre 2000 et 2007) puis les conventions Stations Durables à partir de 2010). Le 3 février 2010 a été signée la première convention Stations Durables de Rhône-Alpes avec Praz-sur-Arly, Cordon et Combloux.

\footnotetext{
${ }^{8}$ La loi NOTRe est à l'origine d'une importante réforme territoriale en France visant la baisse des dépenses publiques et une meilleure prise en compte des besoins des citoyens. Elle s'inscrit dans un mouvement de décentralisation et d'ajustement de la compétence juridique aux réalités géographiques. Elle se traduit notamment par une diminution du nombre de régions françaises (passant de 22 à 13) qui se voient attribuées de nouvelles compétences. En matière de tourisme, le renforcement des intercommunalités opéré par la loi NOTRe est porteur d'une remise en cause de l'exercice de cette compétence au niveau communal. En effet, les communautés de communes et les communautés d'agglomération se voient transférer de nouvelles compétences obligatoires en matière de promotion du tourisme.
} 


\section{Présentation des trois communes ${ }^{9}$}

Combloux est une commune de 17,27 km² avec 2184 habitants et 12800 lits touristiques. Ses remontées mécaniques sont gérées par la SEM (Société d’Economie Mixte) du Jaillet. C'est une station qui se veut «un mélange d'authenticité et d'élégance [...]; c'est vraiment l'esthétique discrète. Et nos clients sont satisfaits de ça. On a essentiellement une clientèle familiale mais il ne faut pas la réduire à la simple jeune famille avec deux enfants. On a aussi des couples sans enfant qui sont des futures familles, des couples de séniors, notamment nos retraités, qui viennent avec leurs petits-enfants, donc oui on peut dire station familiale mais ça regroupe tout un cadre de clientèles différentes qui attendent des choses différentes » (JH, référente Combloux, 17 avril 2015). Située à côté de Megève, Combloux est la station qui a le positionnement «le plus haut de gamme ».

La commune de Cordon s'étend sur 22.5 km², elle compte 1032 habitants, son nombre de lits touristiques est évalué « entre 3800 et 4000 » (CD, référent Cordon, 22 mai 2015). Ses remontées mécaniques sont gérées par la SEM des téléskis de Sallanches-Cordon. C’est une station qui a un positionnement relativement familial cependant moins qualitatif que Combloux. «C'est un domaine qui est très apprécié par rapport à l'ensoleillement, aux paysages et aux enfants, au ski un peu tranquille » (CD, référent Cordon, 22 mai 2015).

Praz-sur-Arly a une superficie de $22.64 \mathrm{~km}^{2}$. Elle compte 1378 habitants et 11000 lits touristiques. Ses remontées mécaniques sont gérées par le groupe privé Labellemontagne. La commune a un positionnement tourné vers les jeunes familles. Elle dispose d’un « sentier thématique au sommet du télésiège associé avec une bande dessinée [...]. C'est une vraie histoire sur les contrebandiers »(PB, $1^{\mathrm{er}}$ Adjoint Praz-sur-Arly, 22 mai 2015). Dans sa

\footnotetext{
${ }^{9}$ Comme précisé plus loin, les verbatim ont été recueillis lors des entretiens menés auprès des acteurs de la mairie des trois stations. Nous avons notamment interrogé les trois « référents », i.e. les personnes responsables pour les trois communes de la mise en place de la charte. Par souci de confidentialité, nous ne donnons ici que les initiales des noms de ces acteurs. Les dates fournies sont celles du recueil des données.
} 
stratégie de diversification quatre saisons, la station se positionne sur le marché des jeunes familles.

Ces trois stations font partie de la Communauté de Communes Pays du Mont-Blanc ${ }^{10}$ depuis le 1er janvier 2013 (année durant laquelle le projet de charte a été finalisé). Elles sont proches géographiquement parlant, bien qu'elles ne partagent pas toutes des frontières communes. En fait, «Combloux et Praz-sur-Arly sont mitoyens par l'altitude » et « il y a des points de rencontre avec Cordon, Combloux et puis avec Praz-sur-Arly » (CD, référent Cordon, 22 mai 2015).

L’espace géographique est ici porteur de représentations communes, renforcées par le constat que les stations ont des tailles similaires et rencontrent les mêmes problématiques étéhiver (manque de neige dû à l’altitude, lits froids importants, concurrence de Megève, etc.). Il est aussi source d'une potentielle confrontation, avec la concurrence latente sur le ski et l’après-ski, avec des clientèles proches, voire communes.

\section{La Convention Station Durable (CSD) 2010-2012}

Né dans le cadre d’une politique contractuelle avec la région Rhône-Alpes (2007/2013), le projet de diversification touristique quatre saisons et de charte de développement durable a été porté avec le soutien de la région Rhône-Alpes et du FEDER (Programme Opérationnel Compétitivité régionale et emploi 2007/2013) dans le cadre de la Convention Stations Durables Combloux / Cordon / Praz-sur-Arly.

Au mois de janvier 2009, une rencontre a eu lieu entre différents élus du Pays du MontBlanc et le Président de la Région Rhône-Alpes. A l’issue de cette entrevue, il est apparu qu'une station comme Cordon pouvait bénéficier d'aides financières de la Région pour développer des actions destinées à diversifier l’offre touristique dans le cadre d'un

\footnotetext{
${ }^{10}$ Cette communauté regroupe au total 10 communes pour coordonner leurs actions dans différents domaines tels que la promotion touristique, les transports scolaires, la collecte des déchets, les aires des gens du voyage, le sport, l'action sociale ou encore le logement (cf. http://www.ccpmb.fr/)
} 
développement durable sur les quatre saisons. Avec Praz sur Arly et Combloux, seules stations éligibles du canton, a été élaboré un projet alliant des actions propres à chaque commune et des actions intercommunales. Cette convention de stations durables a été signée à Cordon en février 2010 pour 2010-2013. Avec l’aide financière de la Région Rhône-Alpes (de $30 \%$ à $60 \%$ selon les actions), et celle de l’Union Européenne dans le cadre du dispositif «Espace Valléen» (jusqu’à 80\% selon les actions), un programme a été établi pour financer les actions retenues.

Les maires et les élus des trois communes ont travaillé ensemble pour mener un programme d'actions reprenant les mêmes fils conducteurs: à ce stade amont de la préparation et jusqu’au lancement de la convention, aucun autre acteur n’avait été associé, les acteurs publics ayant pris la responsabilité de la soumission du projet aux instances de financement. Le caractère incitatif de la convention est à l'origine d'une intention commune qui se limite toutefois, à cette étape, aux seuls maires. "C’est un des grands points forts de ces contrats : inciter les municipalités à travailler en intercommunalité, à mobiliser leurs forces autour de thèmes ou de projets et, enfin, à partager leurs expériences » (YJ, maire de Praz-sur-Arly, 28 mai 2015).

\section{La charte de développement durable}

Les actions (au nombre de huit) prévues dans la charte et leur niveau de réalisation peuvent être visualisés dans la Figure 1 ci-après.

\section{[Insérer ici Figure 1]}

\section{Méthodologie}

Cette recherche est basée sur une méthodologie qualitative qui s'avère particulièrement adaptée pour explorer une question nouvelle ou encore peu étudiée de la littérature (Hlady Rispal, 2002), comme celle traitée dans cet article portant sur la construction des proximités 
institutionnelles. Le choix de recourir à un cas unique composé de trois stations de montagne est justifié au regard de la richesse des données collectées qui permet de saisir la complexité du phénomène étudié à l'aide de «descriptions denses et pénétrantes, nichées dans un contexte réel » (Miles et Huberman, 2003, p. 27).

Notre recherche qualitative exploratoire nous a conduits à interroger les acteurs clés des trois stations ainsi que les deux responsables de l'agence de conseils AIR, le bureau d'étude qui les a accompagnées dans leur démarche. Nous avons réalisé 20 entretiens rétrospectifs d’une durée moyenne de 2 heures entre février et mai 2015 : 4 entretiens avec les deux responsables de l'agence AIR, 3 avec les maires, 6 avec les responsables de l'élaboration de la charte des mairies de Combloux, Cordon, et Praz-sur-Arly et 7 avec divers acteurs impliqués dans le projet représentant le monde socio-économique et civil ${ }^{11}$. Sur les quatre sphères des stations de montagne (économique, politique, civile et clientèle) (Gerbaux et Marcelpoil, 2006), seule la clientèle n’est pas représentée. La sphère civile ne l'est que par une association de propriétaires ${ }^{12}$. Nous avons, pour le choix des personnes interrogées et des entretiens menés, respecté la représentation des sphères impliquées dans le projet d’élaboration de la charte $^{13}$.

L’objectif des entretiens a été de comprendre le processus d'élaboration et de réalisation du contrat, et notamment de la charte qui a été la réalisation principale, et son rôle sur la construction des proximités entre les acteurs. Plus précisément, différents thèmes ont été abordés à l'aide du guide d'entretien semi-directif : 1) Le contexte d'émergence du processus de collaboration entre les trois stations (éléments déclencheurs, modalités d’interaction, choix de la charte), 2) la description du processus de mise en place de la charte (raisons, leviers,

11 Nous ne pouvons fournir de tableau détaillé avec la liste des répondants afin de respecter notre engagement de confidentialité auprès des acteurs interrogés faisant partie du comité de pilotage et du réseau ressources et autres que les trois maires et les responsables de l'élaboration de la charte des trois communes.

12 Cette association des propriétaires se situe à Praz-sur-Arly ; elle n'existe pas dans les deux autres communes. 13 Notons que cette représentation des différentes sphères dans une démarche menée par les mairies est une première au niveau local pour chaque commune, ainsi qu'au niveau du territoire. 
freins, acteurs impliqués et 3) les actions concrètes et les effets associés à la démarche de charte (différences avant/après charte à l'échelle des stations et inter-stations). Parallèlement, nous avons analysé un corpus important de données secondaires : le cahier des charges établi par les trois communes au démarrage du projet, l'ensemble des retours trimestriels de questionnaires envoyés aux acteurs par le bureau d'études (soit 52 questionnaires), les listes du Réseau Ressources et du Comité de Pilotage ${ }^{14}$, les 12 comptes rendus de toutes les réunions, les 18 fiches des diverses actions de la charte, et le bilan 2013 de la charte. L’annexe fournit une frise du déroulement de la mise en place de la charte.

L’analyse des données primaires a été effectuée par codage thématique de manière manuelle. Les codes sont basés sur des catégories issues d’une part, de la littérature institutionnaliste de la proximité pour identifier les différents types de proximités (géographique, institutionnelle, organisationnelle) et d'autre part, de l'approche des objetsfrontières (caractéristiques et propriétés de la charte) pour cerner la contribution de la charte à la construction des proximités nécessaires à la mise en place d’une gouvernance territoriale.

\section{RESULTATS}

\section{Un contexte incitatif favorable mais insuffisant pour engager une action collective}

Le projet de la charte de développement durable a tout d’abord fait émerger la nécessité de conduire une action collective entre les trois stations qui n'étaient jamais entrées en coopération auparavant. L’élément déclencheur a été le soutien financier de la région RhôneAlpes : «C'est souvent la carotte financière qui attire en premier nos élus pour travailler ensemble » (JH, référente Combloux, 17 avril 2015). Les subventions sont vues comme le « levier déclencheur » (CD, référent Cordon, 22 mai 2015) pour la mise en place de la charte

\footnotetext{
${ }^{14}$ Le Réseau Ressources se composait de représentants des communes et d'acteurs privés (mairies, remontées mécaniques, domaines skiables, offices de tourisme, groupements pastoraux, écoles de ski, groupements hôteliers, centre équestre, hôteliers/hébergeurs, commerçants, socio-professionnels, agriculteurs, société de pêche, associations environnementales, accompagnateurs). Le Comité de Pilotage, quant à lui, était formé par des représentants politiques et techniques des trois communes ainsi que des représentants du monde économique: offices de tourisme, hôteliers restaurateurs, commerçants, agriculteurs et remontées mécaniques.
} 
et donc la décision d'entrer dans ce projet. Les trois maires, qui se connaissaient déjà, se sont donc coordonnés pour répondre à cet appel d’offres lancé par la Région : «C’est une volonté de maires justement de pouvoir bénéficier de l'aide financière » $\left(\mathrm{PB}, 1^{\mathrm{er}}\right.$ adjoint Praz-surArly, 22 mai 2015). Les dispositifs publics ont donc joué un rôle clé en fournissant un premier cadre aux maires au service d'une finalité : conduire une action collective à l'échelle des trois stations. Les communes ont pris conscience de la nécessité de passer d'une « situation de concurrence » à la « mutualisation » (Boîte à Outils - Animation, compte-rendu 2012-9-10). Toutefois, si cette incitation était primordiale au démarrage pour convaincre les maires d'entrer en coopération, elle n'était pas suffisante pour que l'ensemble des acteurs des stations puissent interagir et pour réaliser la charte.

D'une part, cette incitation perdait de sa force pour certains acteurs, notamment économiques, qui ne voyaient pas directement l’intérêt de la démarche. D’autre part, seul un partenariat institutionnel avait été initié à l'échelle des trois communes sur des thématiques spécifiques comme la gestion communale des déchets ménagers avec le SITOM (Syndicat Intercommunal de Traitement des Ordures Ménagères) des Vallées du Mont-Blanc dont l’origine remonte à 1969. Il y avait également eu un autre dispositif entre Combloux, Cordon et Sallanches entre 2004 et 2007 (Contrat Plan Interrégional) «mais les actions effectuées étaient propres à chaque commune » (Boîte à Outils - Animation, compte-rendu 2012-9-10)

Ce premier Contrat Stations Durables marque ainsi le début de l'intercommunalité sur des questions autres et est un «projet co-construit au niveau du territoire » (cf. Etude préalable à l'implication des 3 stations touristiques dans une charte de développement durable). Au-delà des acteurs de la sphère publique, les relations entre les acteurs des trois stations restaient largement régies par le mode de l’indifférence ou de la confrontation. 
La charte, un support à la coopération entre acteurs hétérogènes

La charte a été construite à partir d'un cahier des charges réalisé par les techniciens des mairies en décembre 2011 et a "pour objet la réalisation d'une étude environnementale située sur les communes de Cordon, Praz-sur-Arly et Combloux et dont la finalité est de proposer un plan d'action chiffré et phasé [...] s'intégrant dans le cadre de la Charte Nationale en faveur du Développement Durable des Stations de Montagne (...) et s'attachant à créer des synergies et des actions communes entre les trois stations » (Cahier des charges étude préalable, décembre 2011). Cela a nécessité de «consulter les acteurs communaux (personnels et élus des mairies, des domaines skiables et des offices de tourisme) pour mieux comprendre le fonctionnement des structures en place » (Cahier des charges - étude préalable, décembre 2011). Cette large consultation a été soutenue par l'intervention d'un bureau d'études ${ }^{15}$ qui a impulsé une structuration de la démarche dès l'obtention des subventions et le démarrage : «le principal élément, c’est le Réseau Ressources qui a été mis en place. Cela a été insufflé largement par AIR, de créer une synergie qui nous a permis de sortir de notre petit cercle élus-techniciens et d'associer les socio-professionnels, les syndicats divers du territoire, et d'avoir un apport d'idées et un échange avec un cercle plus élargi. Cela a été, je pense, le point le plus important de ce système » (maire de Praz-sur-Arly, 22 mai 2015).

Le but du bureau d'études était de rassembler les acteurs des trois stations afin qu'ils ébauchent ensemble les actions qu'ils avaient envie de porter collectivement et les problématiques qu'ils partageaient sur le territoire. Le bureau d'études a permis à l'objetfrontière de jouer pleinement son rôle de traduction en fixant les rôles de chacun dans le cadre de la création d'un réseau dans lequel les parties agissent ensemble (i.e. le Réseau Ressources et le Comité de Pilotage) et de hiérarchiser les problématiques à traiter. Sans nier certains

\footnotetext{
${ }^{15}$ Au départ AZIMUT et Mountain Riders, qui ont fusionné pour devenir l'agence AIR.
} 
désaccords, cela a conduit à estomper les divergences initiales et à converger vers une représentation partagée. C’est sans doute pour cette raison qu'aucune différence notable n’a pu être observée entre les différentes sphères, voire même entre les divers répondants, selon leurs attributs et fonctions.

Aussi, dès le démarrage, le cahier des charges a été conçu pour prendre en compte l'avis d’une large diversité d’acteurs. De l’avis des répondants, la charte « a appris à décloisonner ; c'est vraiment un des premiers enseignements, à ne pas rester dans des sphères uniquement politiques ou de collectivité mais vraiment se concerter en fait (...). Cela a permis de se rendre compte qu'on partageait un même état des lieux parce que, souvent, on ne travaille qu'à l'échelle de notre commune. On n'a pas forcément conscience qu'on partage les mêmes problématiques et donc, derrière, cela permet de réfléchir à des solutions de manière collective ; c'est vraiment ce processus-là qui s'est mis en route » (JH, référente Combloux, 17 avril 2015).

La charte, un support abstrait, standardisé, modulaire et polyvalent

Cette charte a conduit à rapprocher des acteurs qui étaient géographiquement proches mais qui ne se côtoyaient pas, à l'exception des maires, en créant un cadre suffisamment général qui leur a permis de se coordonner sans renoncer à leurs différences et de s’approprier des ressources pour mener à bien des actions individuelles et collectives au sein d'un espace partagé. Par le biais du questionnaire et des réunions, les acteurs (élus, mairies, offices du tourisme, agriculteurs, hébergeurs, restaurateurs, etc.) ont fait ressortir leurs priorités, ce qui a permis d’élaborer des fiches actions à mener, chacune étant pilotée par un chef de file.

L'abstraction de l'objet-frontière au départ a facilité le dialogue et a permis une standardisation de l'information interprétable par chaque chef de file. Par exemple, le chef de file en charge de l'accessibilité devait « recenser le nombre de personnes à former au sein des OT/Communes » (Compte-rendu du 5ème petit déjeuner du réseau, 27 mai 2013). Le chef de 
file traitant de la problématique des déchets avait pour mission de «lancer une campagne de communication vers les professionnels des métiers de bouche pour faire connaître les possibilité de formation, de diagnostic et d'accompagnement par le SITOM » (Compte-rendu du 5ème petit déjeuner du réseau, 27 mai 2013).

Divers projets ont émergé de cette charte de développement durable car celle-ci était «malléable» (maire de Combloux, 17 avril 2015). Au niveau des actions individuelles, citons le plan d'eau Biotope de Combloux (une piscine écologique), un itinéraire pédestre à Praz-surArly et la construction d'un chalet d'accueil à Cordon. A l'échelle de Combloux et Cordon, les offices de tourisme ont acquis des vélos à assistance électrique pour les saisons d'été. Et plusieurs actions ont été effectuées à l'échelle des trois stations comme la gestion des déchets, la création d'une carte d'accueil commune ainsi que la formation des offices du tourisme et des services touristiques sur l'accessibilité touristique. Nous retrouvons ici la caractéristique de modularité de l'objet-frontière dont les différentes parties (ici, les actions) ont servi de base de dialogue entre les acteurs mais aussi celle de polyvalence dans la mesure où plusieurs actions ont été possibles. En outre, les réunions ont permis de réaliser les actions de la charte. Les participants avaient élaboré huit fiches-actions à l'échelle inter-stations. Au final, cinq actions sur les huit ont été engagées (cf. figure 1) ; une (encourager l'activité agricole, demandée principalement par les agriculteurs) a été identifiée, mais n’a pas abouti car elle dépassait l’échelle des trois communes. Deux autres n’ont pas débuté (création de stations de lavage écologique pour les VTT, et action de concertation avec les transporteurs pour améliorer l'offre de transports en commun) car ce sont des «problématiques qui ne se traitent pas forcément à trois en fait donc, ces actions-là, elles ont été soulevées mais elles ont été portées à la connaissance de la Communauté de Communes des Pays du Mont-Blanc »(JH, référente Combloux, 17 avril 2015). 
La charte, un objet-frontière créateur d'une proximité institutionnelle

La nature suffisamment générale de la charte (abstraction), structurée (standardisation) et suffisamment malléable (modularité et polyvalence) a permis de faire émerger une proximité institutionnelle entre les acteurs au travers d'un large processus de concertation et de négociation. Ils ont pu adapter leurs actions à leur spécificité tout en contribuant à l’objectif commun à l'échelle des trois stations et à la création d'un espace identitaire compte tenu de la " flexibilité interprétative » de l’objet-frontière. Toutefois, cette flexibilité interprétative n’aurait, semble-t-il, pas pu être mise en œuvre sans une infrastructure à travers la fixation de règles et de rôles précis dans le cadre du réseau ressources et du comité de pilotage. Sans cela, un point de vue dominant aurait pu s'imposer aux autres. Le rôle régulateur de l'objetfrontière a joué en positionnant les acteurs les uns par rapport aux autres, de manière complémentaire, pour atteindre un objectif commun.

\section{DISCUSSION}

La charte a permis de produire du sens (dimension cognitive) et de faire des choix (dimension politique) entre des mondes différents (les trois stations mais aussi à l'intérieur d’une même station), socles de la création d’une proximité institutionnelle. Ce résultat a pu être atteint, car bien qu'initiée dans le cadre de la politique régionale, elle n’a jamais été imposée aux acteurs et apparaît bien comme le fruit d’une intentionnalité collective.

Au niveau théorique, le principal apport de cette recherche réside dans la manière dont il est possible de transposer le concept d'objet-frontière pour participer à la construction d'une approche dynamique des proximités. L'introduction de l'objet-frontière en fournit une première étape en éclairant les mécanismes (cognitifs et politiques) de construction de la proximité institutionnelle. Notre recherche montre tout d'abord que la réussite de l'objetfrontière à coordonner des acteurs sur un territoire en cours de construction et à créer une proximité institutionnelle tient au fait qu'il permet « tout autant la construction d'un espace 
de sens commun que d'un espace de hiérarchisation, de choix et de régulation collective » (Talbot, 2008, p. 295). Parce que qu'il est suffisamment commun et malléable, l'objetfrontière a permis en effet de créer une communauté de sens qui faisait défaut tout en garantissant une autonomie de fonctionnement dans un cadre établi collectivement. Certes, le lien entre objet-frontière et proximité institutionnelle n’aurait pas été possible sans incitation à la coopération. A ce titre, l’intervention des pouvoirs publics a joué un rôle de déclencheur clé grâce à l'apport de ressources financières. Par ailleurs, ce dispositif a pu permettre aux stations de prendre conscience que cette collaboration pouvait être une force pour leur territoire en tant que « destination touristique » (terme repris de Bornhorst et al., 2010).

Cette recherche montre aussi que la dynamique observée ne répond pas à un simple processus de mise en conformité des actions individuelles à un cadre imposé. Les acteurs ont su s’approprier le cadre règlementaire et construire leurs propres règles d’interaction au service d'une finalité commune grâce à l'objet-frontière. Ce dernier ne doit pas être vu comme un dispositif d'alignement des représentations des acteurs visant à les rendre semblables. Parce qu’il permet de maintenir la tension entre coopération et hétérogénéité (Trompette et Vinck, 2009), il favorise les interactions futures entre les acteurs tout en maintenant leurs différences. L'objet-frontière participe donc à la différenciation et à l'intégration des acteurs et transforme leurs frontières (Carlile, 2004) en limitant les risques de lock-in dus à un excès de proximité (Boschma, 2005), ouvrant ainsi la voie à une gouvernance territoriale. Cela n’annule pas l'émergence de potentiels désaccords, voire de conflits, mais le cadre ainsi construit permet de les résoudre plus rapidement et d'atteindre une solution satisfaisante pour tous. Ainsi, en dépit d’une diversité élevée entre acteurs, l'objet frontière, animé par un acteur neutre, a permis d'identifier et de résoudre les divergences rapidement (Iorio et Taylor, 2013). 
Cette recherche s’est par ailleurs attachée à montrer le rôle clé joué par l’objet-frontière pour construire la proximité institutionnelle nécessaire à la mise en place de la gouvernance territoriale, qui renvoie à la nécessité d’intégrer la diversité des acteurs à différents échelons décisionnels, que ce soit au niveau local ou régional - voire à une plus large échelle, où se créent différents modes de management touristique des stations (François et Marcelpoil, 2008).

Le territoire est donc né à partir de la révélation d'un problème commun qui a été solutionné par l'objet-frontière. Une question qui reste posée est celle de la pérennité du processus engagé et de la création d'une proximité organisationnelle entre les stations. Nos données ne nous permettent pas d’avoir le recul suffisant pour évaluer le rôle de l'objetfrontière dans l'établissement d'un « lieu [pérenne] de production et d'activation des règles et routines qui permettent de prendre des décisions au nom des acteurs » (Talbot, 2008, p. 302). Il ressort de notre analyse que l’objet-frontière est un levier pour dépasser les limites existantes des modèles traditionnels de gouvernance ainsi que leur expression, centrés sur un (souvent l’opérateur de remontées mécaniques) ou quelques acteurs peu représentatifs des intérêts des différentes sphères économique, politique, civile et clientèle en présence (Gerbaux et Marcelpoil, 2006).

Au niveau empirique, il est intéressant de constater que la politique volontariste ayant favorisé la mise en place de la charte n’a pas uniquement permis la réalisation d’actions concrètes à l'échelle des trois stations mais a également conduit les maires des trois communes à tenter de répondre à un projet à une plus grande échelle : celle de la Communauté des Communes des Pays du Mont-Blanc. Les trois maires ont réussi à convaincre les sept autres communes, grâce à leur projet de charte réussi, d’aller vers une gouvernance territoriale plus large, notamment au niveau touristique. Il y a donc eu un 
élargissement de l’échelle, certaines problématiques dépassant la compétence des trois stations (comme le maintien du foncier agricole).

La Communauté de Communes Pays du Mont-Blanc a candidaté en septembre 2015 pour la convention Espace Valléen ${ }^{16}$, suite « logique » pour que les stations continuent à travailler ensemble : «si on n'avait pas fait cette charte, je ne pense pas qu'on serait passé sur un nouveau contrat Espace Valléen à dix, parce que c'est cette charte qui a permis de ne pas se focaliser uniquement sur les actions communales mais bien de montrer qu'il y avait un intérêt collectif à réfléchir au développement touristique » (JH, référente Combloux, 17 avril 2015).

Ainsi le démarrage de la gouvernance à trois a favorisé l'éventuel élargissement de la gouvernance à dix en montrant qu'il était opportun de s’affranchir des frontières géographiques au niveau de la commune et de réaliser des actions conjointes au niveau d’un territoire.

Toutefois, au travers des compositions du Réseau Ressources et du Comité de Pilotage, la sphère civile n'est présente que dans une station (Praz-sur-Arly) au travers d'une seule association (Bien Vivre à Praz-sur-Arly). La gouvernance territoriale pourrait donc être améliorée en impliquant davantage les civils (citoyens et touristes). Cet élargissement nous semble d'autant plus important que les stations sont aujourd'hui confrontées au problème des lits froids, dans lequel ils sont impliqués. La présence de l'ensemble des sphères s’avère donc essentielle (Gerbaux et Marcelpoil 2006). En outre, la sphère politique doit prendre en compte les attentes de la société civile pour répondre à l'ensemble de ses attentes sur sa qualité de vie et de son intérêt pour préserver son environnement. Le tableau 2 ci-après fournit une lecture des changements observés après l'élaboration et la mise en place de la charte.

\section{[Insérer ici Tableau 2]}

\footnotetext{
$\cdot{ }^{16}$ Cf. http://www.ccpmb.fr/les-projets-pour-le-territoire/contrats-regionaux-et-europeens/les-projets-europeens.
} 
Au plan managérial, la mise en place de la charte via l'objet-frontière peut être rapprochée de la gestion de projet ${ }^{17}$. En effet, la littérature en gestion de projet reconnaît très largement les avantages associés à l'implication de l'ensemble des parties prenantes au cours du projet tout en soulignant l'importance de mobiliser des objets-frontières pour créer une connaissance et un savoir commun (Koskinen et Mäkinen, 2009). Pour cela, l'objet-frontière permet d'atteindre les différentes étapes nécessaires au passage d’une connaissance dispersée et diverse entre les acteurs du territoire à une connaissance commune sur les véritables enjeux et la manière de développer le projet (Di Marco et al., 2012).

Dans ce cadre, des techniques de visualisation du savoir ou des méthodes utilisées par les créatifs pour faire émerger des idées peuvent être mobilisées dès la phase amont du projet pour négocier le contrat. Ces objets-frontières facilitent le partage du savoir et des connaissances qui, à cette étape, ne sont seulement explicites (mémos, notes, documents, plans, instructions, etc.) mais aussi formées d'opinions subjectives, d'intuitions et d’impressions des acteurs. Par ailleurs, compte tenu de la forte hétérogénéité des acteurs en présence lors des négociations contractuelles (que ce soit avec les institutions publiques ou avec les autres acteurs territoriaux), les incompréhensions sont fréquentes. Des objetsfrontières institutionnalisés comme les mémos, dessins et représentations graphiques ou noninstitutionnalisés comme des événements (rencontres informelles, petits déjeuners, etc.) favorisant l'ouverture, la confiance et le travail commun s'avèrent pertinents. Enfin, des prototypes, miniaturisations, représentations graphiques, dessins ou plus encore des objets 3D peuvent être développés afin d’avoir une vision commune des éléments concrets et tangibles qui vont être réalisés pour le projet (Lehmann, Rousseau, 2016). Par exemple, une cartographie des routes accessibles pour le sous-projet vélo électrique de la charte de développement durable aurait pu permettre d'éviter des difficultés à visualiser l'étendue du dispositif et les possibilités d'accès pour ce type de vélo. De la même manière, une carte

\footnotetext{
${ }^{17}$ Nous remercions l'un des évaluateurs pour cette suggestion.
} 
visualisant les différents types de terrain agricole auraient pu être utilisée, ainsi que des dessins des diverses activités agricoles pour favoriser le dialogue avec les agriculteurs du territoire et encourager l'activité agricole. Cette action concernant l'aménagement du territoire n’a pas été engagée - alors qu’elle avait été prévue initialement dans la charte, notamment du fait de l'étendue de son périmètre au-delà des trois stations mais aussi de représentations opposées entre les acteurs publics et les agriculteurs. Pour gérer le projet, les timelines (comme les schémas de Gantt) comme objets-frontières temporels peuvent être également très utiles, notamment pour retracer les étapes clés du projet et les deadlines. Cette représentation graphique et visuelle permet aux acteurs de créer du sens et une compréhension commune du projet (Chang et al., 2013).

Au-delà de la portée de l'objet-frontière, cette recherche montre aussi le rôle joué par le chef de projet, endossé ici par un cabinet de conseil, qui a su associer et impliquer les divers acteurs en leur faisant comprendre la portée du projet. Ce rôle de chef de projet est délicat et exige audace et prudence (Lehmann et Rousseau, 2016). Le choix de recourir à un bureau d'étude en position de neutralité s'est avéré judicieux dans un contexte marqué par un déficit de compétences managériales.

\section{Conclusion}

Cette recherche débouche sur deux résultats principaux. Premièrement, nous mettons en avant le fait que les soutiens et dispositifs publics mis en place pour l'intercommunalité jouent certes un rôle incitatif mais qui reste insuffisant pour engager un processus collaboratif entre les trois stations. Deuxièmement, nous montrons l'importance de la charte en tant qu'objetfrontière pour créer une proximité institutionnelle donnant la possibilité à une gouvernance territoriale d'émerger. Au plan cognitif, l'objet-frontière a permis la construction d'une représentation commune et partagée entre des acteurs hétérogènes (et qui n’avaient pas du tout l'habitude de se côtoyer). Au plan politique, elle a conduit à la formation d'un espace de 
régulation qui permet non seulement d'éviter des effets d'enfermement (lock in) mais surtout à redéfinir collectivement les frontières de la gouvernance territoriale autour d'actions partagées à l’échelle inter-stations.

L’ensemble des éléments précités permet de fournir un certain nombre de recommandations pour les pouvoirs publics en charge de l'élaboration des politiques régionales, notamment en territoires de montagne. A l'heure des Communautés de Communes (CC) entre communes parfois très distantes, non seulement au niveau géographique, mais aussi aux niveaux cognitif, organisationnel et institutionnel ${ }^{18}$, les pouvoirs publics représentent clairement des leviers pour la création des proximités nécessaires à la mise en place de la gouvernance territoriale grâce à leurs politiques volontaristes de configuration. Ils peuvent susciter des territoires aux regroupements pertinents à condition que ces derniers ne soient pas imposés dans un cadre trop contraignant et laissent aux acteurs impliqués le temps et les moyens nécessaires pour construire leurs propres règles de fonctionnement. Cette recherche est de nature également à éclairer les leviers mobilisables par les acteurs des stations de montagne en France comme à l'étranger.

Des travaux ont montré que bien des stations à l'étranger sont confrontées à des problèmes similaires. Crans-Montana-Aminona en Suisse fournit un cas exemplaire de station qui ne parvient pas à engager un partenariat stratégique entre les différents acteurs afin d'assurer une « bonne » gouvernance du système touristique à l'échelle territoriale (Clivaz, 2006). D’autres études font des constats similaires en Suède (Nordin, et Svensson, 2007) ou encore en Italie (D’Angella et al., 2010).

De manière générale, il convient de renforcer les connaissances quant à la manière de développer les proximités sous-jacentes à la gouvernance territoriale. Des recherches futures

\footnotetext{
${ }^{18} \mathrm{Cf}$. ces CC réunissant à la fois des communes en vallée et des stations de sport d'hiver, souvent de tailles et cultures très variées, comme la CC des Pays du Mont-Blanc.
} 
pourraient s'intéresser à l'observation de cas d'échecs de gouvernance territoriale pour enrichir l’approche institutionnaliste des proximités. 


\section{Bibliographie}

Achin, Coralie (2015). La gouvernance de la diversification comme enjeu de l'adaptation des stations de moyenne montagne : L'analyse des stations de la Bresse, du Dévoluy et du Sancy, Thèse de Doctorat, Université Grenoble Alpes.

Association Nationale des Elus de Montagne (2013). De nouvelles pistes pour les stations de moyenne montagne, dossier $n^{\circ} 234$, janvier.

Balland, Pierre-Alexandre; Boschma, Ron; Frenken, Koen (2015). Proximity and Innovation: From Statics to Dynamics, Regional Studies, Vol. 49, n6, p. 907-920.

Berry, Michel (1983). Une technologie invisible - L'impact des instruments de gestion sur l'évolution des systèmes humains. CRG-1133. Cahier du laboratoire.

Bocquet, Rachel (2008). Proximités et coordination inter-firmes: une analyse empirique de la compétitivité des stations de sports d'hiver, Revue d'Economie Régionale et Urbaine, Vol. 2, p. 159-178.

Bornhorst, Tom; Brent Ritchie, JR, Sheehan, Lorn (2010). Determinants of tourism success for DMOs \& destinations: An empirical examination of stakeholders’ perspectives, Tourism Management, Vol. 31, n5, p. 572-589.

Boschma, Ron (2005). Proximity and Innovation: A critical assessment, Regional Studies, Vol. 39, n²1, p. 61-74.

Boudières, Vincent; Marcelpoil, Emmanuelle (2006). Gouvernance touristique des grandes stations et durabilité. Une lecture en termes de proximité, Développement durable et territoires : http://developpementdurable.revues.org/2648. 
Callon, Michel (1986). Eléments pour une sociologie de la traduction. La domestication des coquilles Saint Jacques et des marins-pêcheurs dans la baie de Saint-Brieuc, L'année sociologique, Vol. 36, p. 169-208.

Carlile, Paul (2004). Transferring, Translating, and Transforming: An Integrative Framework for Managing Knowledge Across Boundaries, Organization Science, Vol. 15, n5, p. 555-568. Chang, Artemis; Hatcher, Caroline ; Kim, Jai (2013). Temporal boundary objects in megaprojects: Mapping the system with the Integrated Master Schedule, International Journal of Project Management, Vol. 31, p. 323-332.

Clivaz, Christophe (2006). Crans-Montana-Aminona (Suisse) : y a-t-il un pilote dans la station ?, Revue de géographie alpine, Vol 94, n²1, p. 75-83.

Colletis, Gabriel ; Pecqueur, Bernard (2005). Révélation de ressources spécifiques et coordination située, Economie et Institutions, Vol. 6-7, p. 51-74.

D’Angella, Francesca ; De Carlo, Manuella ; Sainaghi, Ruggero (2010). Archetypes of destination governance: A comparison of international destinations, Tourism Review, Vol. 65, n 4 , p. 61-73.

Di Marco, Melissa; Alin, Pauli ; Taylor, John (2012). Exploring Negotiation Through Boundary Objects in Global Design Project Networks, Project Management Journal, Vol. 43, n³, p. 24-39.

Gerbaux, Françoise; Marcelpoil, Emmanuelle (2006). Gouvernance des stations de montagne en France: les spécificités du partenariat public-privé, Revue de géographie alpine, Vol. 94, n¹, p. 9-19.

Gilly, Jean-Pierre; Wallet, Frédéric (2005). Enchevêtrement des espaces de régulation et gouvernance territoriale. Les processus d'innovation institutionnelle dans la politique des Pays en France, Revue d'Économie Régionale et Urbaine, Vol. 5, p. 699-722. 
Gilly, Jean-Pierre ; Torre, André (2000). Dynamiques de Proximité, L’Harmattan, Paris.

François, Hugues; Marcelpoil, Emmanuelle (2008). Les processus d'articulation des proximités dans les territoires touristiques. L’exemple des stations de montagne, Revue d'Economie Régionale \& Urbaine, Vol. 2, p. 179-191.

Hlady Rispal, Martine (2002). La méthode des cas: Application à la recherche en gestion. De Boeck Supérieur, 256 pages.

Iorio, Josh ; Taylor, John (2014). Boundary object efficacy: The mediating role of boundary objects on task conflict in global virtual project networks, International Journal of Project Management, Vol. 32, p. 7-17.

Koskinen, Kaj ; Mäkinen, Seppo ( 2009). Role of boundary objects in negotiations of project contracts, International Journal of Project Management, Vol. 27, p. 31-38.

Leloup, Fabienne ; Moyart, Laurence ; Pecqueur, Bernard (2005). La gouvernance territoriale comme nouveau mode de coordination territoriale ?, Géographie Economie Société, Vol. 7, n4, p. 321-332.

Lehmann, Valérie; Rousseau, Frédéric (2016). 3D boundary objects in stakholder management : knowledge creators for the project and collaboration facilitators, The Journal of Modern Project Management, Vol. 3 (3), p. 105-111

Marcelpoil, Emmanuelle (2012). Gouvernance des stations : Etat des lieux et perspectives, Juristourisme, Vol. 140, p. 32-34.

Miles, Matthew B., A; Huberman Michael (2003). Analyse des données qualitatives, De Boeck Supérieur, 632 pages. 
Nordin, Sara. ; Svensson Bo (2007). Innovative destination governance: The Swedish ski resort of Åre, International Journal of Entrepreneurship and Innovation, Vol. 8, n 1 , p. 5366.

Star, Susan Leigh (1999). The ethnography of infrastructure, American Behavioral Scientist, Vol. 43, n³, p. 377-391.

Star, Susan Leigh; Griesemer, James R (1989). Institutional Ecology, «Translations » and Boundary Objects: Amateurs and Professionals in Berkeley’s Museum of Vertebrate Zoology, Social Studies of Science, Vol. 19, n³, p. 387-420.

Swan, Jacky; Bresnen, Mike; Newell, Sue; Robertson, Maxine (2007). The object of knowledge: The role of objects in biomedical innovation, Human Relations, Vol. 60, $\mathrm{n}^{\circ} 12$, p.1809-1837.

Swan, Jacky; Scarbrough, Harry; Robertson, Maxine (2002). The Construction of 'Communities of Practice’ in the Management of Innovation, Management Learning, Vol. 33, $\mathrm{n}^{\circ} 4$, p. $477-496$.

Talbot, Damien (2008). Les institutions créatrices de proximités, Revue d'Economie Régionale \& Urbaine, Vol. 3, p. 289-310.

Torre André, (2010), Jalons pour une analyse dynamique des Proximités, Revue d'Économie Régionale \& Urbaine, Vol. 3, p. 409-437.

Torre, André ; Rallet, Alain (2005). Proximity and localization, Regional Studies, Vol. 39, $\mathrm{n}^{\circ} 1$, p. $47-59$.

Trompette, Pascale ; Vinck, Dominique (2009). Retour sur la notion d'objet-frontière, Revue d'anthropologie des connaissances, Vol. 3, n¹, p. 5-27. 
Vinck, Dominique (2009). De l'objet intermédiaire à l'objet-frontière. Vers la prise en compte du travail d’équipement, Revue d'anthropologie des connaissances, Vol. 3, n¹, p. 210-232.

Weedman, Judith (1998). The Structure of Incentive: Design and Client Roles in ApplicationOriented Research, Science Technology Human Values, Vol. 23, n³, p. 315-345.

Wenger, Etienne (2000). Communities of Practice and Social Learning Systems, Organization, Vol. 7, n², p. 225-246.

Zimmerman, Jean-Benoît (2008). Le territoire dans l'analyse économique, Proximité géographique et proximité organisée, Revue Française de Gestion, Vol. 4, n¹84, p. 105-118. 